PROCEEDINGS OF THE

AMERICAN MATHEMATICAL SOCIETY

Volume 141, Number 9, September 2013, Pages 3275-3289

S 0002-9939(2013)11586-8

Article electronically published on May 31, 2013

\title{
LIMITWISE MONOTONIC SEQUENCES AND DEGREE SPECTRA OF STRUCTURES
}

\author{
ISKANDER KALIMULLIN, BAKHADYR KHOUSSAINOV, AND ALEXANDER MELNIKOV
}

(Communicated by Julia Knight)

\begin{abstract}
In this paper, we study effective monotonic approximations of sets and sequences of sets. We show that there is a sequence of sets which has no uniform computable monotonic approximation but has an $\mathbf{x}$-computable monotonic approximation for every hyperimmune degree $\mathbf{x}$. We also construct a $\Sigma_{2}^{0}$ set which is not limitwise monotonic but is $\mathbf{x}$-limitwise monotonic relative to every non-zero $\Delta_{2}^{0}$ degree $\mathbf{x}$. We show that if a sequence of sets is uniformly limitwise monotonic in $\mathbf{x}$ for all except countably many degrees $\mathbf{x}$, then it has to be uniformly limitwise monotonic. Finally, we apply these results to investigate degree spectra of abelian groups, equivalence relations, and $\aleph_{1}$ categorical structures.
\end{abstract}

\section{INTRODUCTION}

This paper is motivated by research on degree spectra of structures from various classes given by algebraic or model-theoretic properties. Examples of such classes of structures include abelian groups, linearly ordered sets, models of $\aleph_{1}$-categorical theories, and equivalence structures. In this paper all structures we consider are countable. Recall the following definition central to this paper:

Definition 1.1. The degree spectrum of a countable structure $\mathcal{A}$, denoted by $\operatorname{DegSp}(\mathcal{A})$, is the collection of all Turing degrees $\mathbf{d}$ such that there exists an isomorphic copy $\mathcal{B}$ of $\mathcal{A}$ with $D_{0}(\mathcal{B}) \equiv_{T} \mathbf{d}$, where $D_{0}(\mathcal{B})$ is the open diagram of $\mathcal{B}$ and $\equiv_{T}$ is the Turing equivalence relation.

Research on degree spectra of structures has been quite active in recent years. Here we mention some of the related results. Slaman [22] proved that there exists a structure $\mathcal{M}$ such that $\mathcal{M}$ has an a-computable copy if and only if $\mathbf{a}>0$ (see [26] for an alternate proof). More generally, for every computable ordinal $\alpha$ there is a structure which has exactly non - $\operatorname{low}_{\alpha}$ degrees serving as its degree spectum (see [10]). Csima and Kalimullin [4] built a structure $\mathcal{H}$ such that $\mathcal{H}$ has an acomputable copy if and only if $\mathbf{a}$ is hyperimmune. Examples of this kind can be found in various natural classes of algebraic structures. For instance, Miller 20]

Received by the editors September 14, 2010 and, in revised form, February 21, 2011, November 7, 2011, and November 30, 2011.

2010 Mathematics Subject Classification. Primary 03C57, 03D75, 03D80.

The first author was partially supported by RFBR grants 09-01-97010, 10-01-00399 and by the Russian President Grant MK-1784.2010.1.

All authors acknowledge support of the Marsden Fund of the Royal Society of New Zealand.

(C) 2013 American Mathematical Society 
built a linear order $L$ such that $\operatorname{Deg} S p(L)$ contains a $\Delta_{2}^{0}$ degree a if and only if a $>$ 0. Similar examples can be found in other classes of structures (see, e.g., [15], [9] and [21]).

The main technical computability-theoretic concept introduced in this paper is the generalization of limitwise monotonicity to sequences of sets. This definition is motivated mainly by our desire to study degree spectra of specific structures such as torsion abelian groups, equivalence structures and models of $\aleph_{1}$-categorical theories.

Recall that a set $S$ is limitwise monotonic relative to a given degree a if there is an a-computable function $g: \omega \times \omega \rightarrow \omega$ such that (1) $\max _{s} g(x, s)$ exists for every $x$, and $(2) S=\operatorname{rng}\left(\lambda x\left[\max _{s} g(x, s)\right]\right)$. The second condition can equivalently be replaced by $\left(2^{\prime}\right) g$ is total, $g(x, s) \leq g(x, s+1)$ for all $x, s \in \omega$, and $\lim _{s} g(x, s)$ exists. We say that the function $f(x)=\max _{s} g(x, s)$ is limitwise monotonic.

Definition 1.2. An ordered sequence of sets $\mathcal{S}=\left\{S_{n}\right\}_{n \in \omega}$ is uniformly limitwise monotonic (relative to $\mathbf{a}$, in $\mathbf{a}$ ) if there is a computable (a-computable) function $g$ such that

- $\max _{x} g(n, y, x)$ exists for every $n, y \in \omega$ and

- $S_{n}=\operatorname{rng}\left(\lambda y\left[\max _{x} g(n, y, x)\right]\right)$ for every $n \in \omega$.

We say that $g$ is a uniform limitwise monotonic (relative to $\mathbf{a}$, in $\mathbf{a}$ ) approximation of $\mathcal{S}$.

This is a clear generalization of the concept of limitwise monotonic sets. It follows that a set $A$ is limitwise monotonic if and only if the sequence $\left\{S_{n}\right\}_{n \in \omega}$, where $S_{n}=A$ for all $n$, is uniformly limitwise monotonic.

Limitwise monotonic sets have been used in computable model theory. Khisamiev was the first to introduce the notions of computable monotonic approximation and the notions of $s$-function and $s_{1}$-function [18. He used computable monotonic approximations of $\Sigma_{2}^{0}$ sets to study computable abelian $p$-groups of small Ulm length [18. He also showed that there is a $\Delta_{2}^{0}$ set which is not limitwise monotonic (Proposition 3.8 of [18]). Independently, Khoussainov, Nies and Shore in [19] introduced limitwise monotonic functions in the study of computable models of $\aleph_{1}$ categorical theories. They gave an alternate construction of a $\Delta_{2}^{0}$-set which is not limitwise monotonic. They used such sets to construct an $\aleph_{1}$-categorical but not $\aleph_{0}$-categorical theory, all of whose countable models apart from the prime model are computable. Csima, Hirschfeldt, Knight and Soare [3] applied limitwise monotonicity to examine the complexity of prime models of theories. Harris [11] used this concept to study computable linear orders. Kach [16] applied limitwise monotonic approximations to shuffle sums of ordinals. Calvert, Cenzer, Harizanov and Morozov [2] studied equivalence structures using limitwise monotonicity. Hirschfeldt, Miller and Podzorov [13] used limitwise monotonic approximations to study ordercomputable sets. They also showed that there is a low set which is not limitwise monotonic. Coles, Downey, and Khoussainov 23 used limitwise monotonic sets in the construction of computable linear orders with $\Pi_{2}^{0}$-initial segments that are not computably presentable. Hirschfeldt [12] used limitwise monotonic sets to exhibit a complete theory of linearly ordered sets with both a computable model and a prime model but with no computable prime model. Khoussainov, Hirschfeldt and Semukhin in [14] used a variation of limitwise monotonicity to build an $\aleph_{1}$-categorical but not $\aleph_{0}$-categorical theory whose only computable model is saturated. Kach 
and Turetsky [17] introduced generalizations of limitwise monotonicity and studied them. Recently, Downey, Kach and Turetsky [6] found a nice connection between limitwise monotonicity and non-highness in c.e. Turing degrees.

In the first part of our paper we study sequences of sets with an eye towards uniform limitwise monotonicity. Our first theorem builds a sequence of sets that is uniformly limitwise monotonic in every hyperimmune degree but which fails to be uniformly limitwise monotonic.

Theorem 1.3. There is a sequence of infinite sets which is uniformly limitwise monotonic relative to every hyperimmune degree (in particular, relative to every non-zero $\Delta_{2}^{0}$-degree) but is not uniformly limitwise monotonic.

The proof of the next theorem uses the ideas of the proof of the theorem above. However, we note that in the theorem above we are able to handle uncountably many (hyperimmune) degrees, while in the theorem below, in the case of a single set, we are able to deal with only countably many degrees.

Theorem 1.4. There is a $\Sigma_{2}^{0}$ set $S$ such that $S$ is limitwise monotonic in every non-zero $\Delta_{2}^{0}$-degree but is not limitwise monotonic.

This theorem also gives us the following corollary:

Corollary 1.5 (Downey, Kach, Turetsky [6], Wallbaum [25]). For every non-zero degree $\mathbf{a}$, there is a set $S$ which is limitwise monotonic in a but is not limitwise monotonic.

Proof of Corollary [1.5. If $\mathbf{a}$ is $\Delta_{2}^{0}$, we can take the set $S$ from Theorem 1.4. If a is not $\Delta_{2}^{0}$, then we can just choose a set $S \in \mathbf{a}$ which is not $\Sigma_{2}^{0}$.

A natural question arises if Theorem 1.3 can be extended to all non-zero degrees. This question is answered in the next theorem:

Theorem 1.6. If a sequence of sets $\left\{S_{n}\right\}_{n \in \omega}$ is uniformly limitwise monotonic in all degrees except perhaps countably many, then $\left\{S_{n}\right\}_{n \in \omega}$ is uniformly limitwise monotonic.

In contrast, Wallbaum 25] has recently shown that there is a $\Delta_{2}^{0}$ set which is not limitwise monotonic but is limitwise monotonic relative to each member of a class of measure one in the Cantor space $2^{\omega}$.

In the second part of our paper we apply the above theorems to specific classes of computable structures. For instance, we apply Theorem 1.3 and Theorem 1.4 to abelian groups and obtain the following result:

\section{Theorem 1.7.}

(1) There is a torsion abelian group $G$ such that (a) $G$ has no computable copy and (b) $G$ has an a-computable copy for every hyperimmune degree a.

(2) There is an abelian p-group $A$ such that $\operatorname{Deg} \operatorname{Sp}(A)$ contains a $\Delta_{2}^{0}$ degree a if and only if $\mathbf{a}>0$. 
In the first part of this theorem, the group $G$ is of the form

$$
G=\bigoplus_{p \in X}\left(\bigoplus_{n \in S_{p}} Z_{p^{n}}\right),
$$

where $X$ is a set of prime numbers and $S_{p} \subseteq \omega$ for each $p \in X$. In the second part, the group $A$ is a $p$-group and is of the form $\bigoplus_{n \in S} Z_{p^{n}}$, where $S \subseteq \omega \backslash\{0\}$.

It is natural to ask whether Theorem 1.7 can be strengthened for groups of the form $G=\bigoplus_{p \in X}\left(\bigoplus_{n \in S_{p}} Z_{p^{n}}\right)$. For instance, one would like to know if there exists a group $G$ of such form such that the group has an x-computable copy if and only if $\mathbf{x}>0$ (or if and only if $\mathbf{x}$ is not $\operatorname{low}_{\mathrm{n}}$ for some $n$ ). We apply Theorem 1.6 to show that such groups do not exist:

Theorem 1.8. For any group $G$ of the form $G=\bigoplus_{p \in X}\left(\bigoplus_{n \in S_{p}} Z_{p^{n}}\right)$, where $X$ is a set of prime numbers and $S_{p} \subseteq \omega \backslash\{0\}$ for each $p \in X$, we have the following: If the group $G$ has an $\mathbf{x}$-computable copy for every degree $\mathbf{x}$, except perhaps countably many, then $G$ has a computable copy.

Then we illustrate similar applications of the computably-theoretic results to equivalence structures and $\aleph_{1}$-categorical theories.

\section{Preliminaries}

Here we prove two properties of sequences of sets. The first property states that uniformly limitwise monotonic sequences of infinite sets possess injective enumerations. This is a uniform statement of the result of Harris [11 for limitwise monotonic sets. The second property gives a necessary and sufficient condition for a uniformly $\Sigma_{2}^{0}$-sequence of sets to be uniformly limitwise monotonic.

Proposition 2.1 (Harris [11]). Suppose $\mathcal{S}=\left\{S_{n}\right\}_{n \in \omega}$ is a uniformly limitwise monotonic sequence of infinite sets. Then there is a limitwise monotonic approximation $g(n, y, x)$ of $\mathcal{S}$ such that $\lambda y\left[\max _{x} g(n, y, x)\right]$ is injective for every $n \in \omega$.

The following proof is a uniform version of the proof for the case of a single set (see [1] or 6]).

Proof. Let $f(n, y, x)$ be a uniform limitwise monotonic approximation for $\mathcal{S}$. Without loss of generality we can assume that $\lambda x[f(n, y, x)]$ is non-decreasing for every $n, y \in \omega$.

First we set $g(n, y, x)=0$ if $x \leq y$. To define $g(n, y, x)$ for $x>y$, suppose that for every $y^{\prime}<y$ and $x^{\prime}<x$ the values of $g\left(n, y^{\prime}, x\right)$ and $g\left(n, y, x^{\prime}\right)$ have already been defined. Choose $\left\langle y_{0}, x_{0}\right\rangle$ least such that $x_{0}>x, f\left(n, y_{0}, x_{0}\right) \geq \max _{x^{\prime}<x} g\left(n, y, x^{\prime}\right)$ and $f\left(n, y_{0}, x_{0}\right) \notin\left\{g\left(n, y^{\prime}, x\right) \mid y^{\prime}<y\right\}$. Set $g(n, y, x)=f\left(n, y_{0}, x_{0}\right)$.

It is not hard to check that $\lambda y\left[\max _{x} g(n, y, x)\right]$ is total and injective. It is also evident that $\operatorname{rng}\left(\lambda y\left[\max _{x} g(n, y, x)\right]\right) \subseteq S_{n}$ for every $n \in \omega$. To see that $S_{n} \subseteq \operatorname{rng}\left(\lambda y\left[\max _{x} g(n, y, x)\right]\right)$, we use an inductive argument. The definition of $g$ may be viewed as a construction, where at each stage we compute the value of $g$ for exactly one new pair of arguments and the value of $f$ for a new pair of arguments as well. We denote our current guess about $\max _{x} g(n, y, x)$ at stage $s$ by $\left[\max _{x} g(n, y, x)\right]_{s}$, and similarly for $f$. Suppose there are $y$ and $n$ such that $\max _{x} f(n, y, x) \notin \operatorname{rng}\left(\lambda y\left[\max _{x} g(n, y, x)\right]\right)$ and $y$ is least with this property. 
Then there should be a stage $s$ such that for every stage $t \geq s$ and every $y^{\prime}<y,\left[\max _{x} f\left(n, y^{\prime}, x\right)\right]_{s}=\left[\max _{x} f\left(n, y^{\prime}, x\right)\right]_{t} \in \operatorname{rng}\left(\lambda y\left[\max _{x} g(n, y, x)\right]\right)$. We may further assume that $s$ satisfies $\left[\max _{x} f(n, y, x)\right]_{s}=\max _{x} f(n, y, x)$. By the definition of $g$, there should be a stage $t_{0} \geq s$ and an argument $y_{0}$ such that $\left[\max _{x^{\prime}<x} g\left(n, y_{0}, x^{\prime}\right)\right]_{t_{0}}=\max _{x} f(n, y, x)$, since we always start with $g(n, y, x)=0$ for $x \leq y$. If there is no stage $t_{1} \geq t_{0}$ and $y_{1}<y_{0}$ such that $\left[\max _{x} g\left(n, y_{1}, x\right)\right]_{t_{1}}=$ $\left[\max _{x} g\left(n, y_{0}, x\right)\right]_{t_{0}}$, then $\max _{x} g\left(n, y_{0}, x\right)=\max _{x} f(n, y, x)$. Therefore there should exist $y_{1}$ and a stage $t_{1}$ such that $\left[\max _{x} g\left(n, y_{1}, x\right)\right]_{t_{1}}=\left[\max _{x} g\left(n, y_{0}, x\right)\right]_{t_{0}}$. We can use the same argument to find $y_{2}$ and $t_{2}$, which play the same role for $y_{1}$ and $t_{1}$ as the latter arguments do for $y$ and $t$. Since $y=y_{0}>y_{1}>\ldots$, we will find the least $y_{i}$ in this sequence. But then $\max _{x} g\left(n, y_{i}, x\right)=\max _{x} f(n, y, x)$, contrary to the hypothesis.

We will need the following lemma which gives a necessary and sufficient condition for a $\Sigma_{2}^{0}$ set to be limitwise monotonic.

Lemma 2.2 (Folklore; see, e.g., [6]). An infinite $\Sigma_{2}^{0}$ set is limitwise monotonic if and only if it contains an infinite limitwise monotonic subset.

Proof. Let $S$ be a $\Sigma_{2}^{0}$ set and $U \subset S$ be limitwise monotonic and infinite. Since $S$ is a $\Sigma_{2}^{0}$ set, there exists a computable function $h$ such that for every $z \in \omega$ we have

$$
z \in S \Longleftrightarrow W_{h(z)} \text { is finite. }
$$

Let $f(y, s)$ be a limitwise monotonic approximation of $U$. We shall define $g$ so that $g$ is a limitwise monotonic approximation of $S$.

Fix a computable list $\left\{\left(z_{k}, y_{k}, s_{k}\right)\right\}_{k \in \omega}$ of all triples with the property that $f\left(y_{k}, s_{k}\right) \geq z_{k}$ for all $k \in \omega$. Define

$$
g(k, s)= \begin{cases}z_{k} & \text { if } W_{h\left(z_{k}\right), s} \subseteq W_{h\left(z_{k}\right), s_{k}}, \\ f\left(y_{k}, s\right) & \text { otherwise. }\end{cases}
$$

We claim that $g$ is a limitwise monotonic approximation of $S$. Indeed, first note that for all $k, s \in \omega$, we have $g(k, s) \leq g(k, s+1)$. It is also easy to see that $\max _{s} g(k, s)$ exists for all $k \in \omega$, as $\max _{s} g(k, s) \leq \max _{s} f(k, s)$.

Assume that $z \in S$. Since $U$ is an infinite set, there exists a $k$ such that $f\left(y_{k}, s_{k}\right) \geq z_{k}$, where $z=z_{k}$ and $W_{h\left(z_{k}\right)}=W_{h\left(z_{k}\right), s_{k}}$. Thus, for all $s$ we have $W_{h\left(z_{k}\right), s} \subseteq W_{h\left(z_{k}\right), s_{k}}$. Therefore $\lim _{s} g(k, s)=z_{k}=z$. Now assume that $z \notin S$. Then the set $W_{h(z)}$ must be infinite. Let $k, s$ be such that $g(k, s)=z$. There is an $s^{\prime}>s$ such that $W_{h\left(z_{k}\right), s^{\prime}} \nsubseteq W_{h\left(z_{k}\right), s_{k}}$. Then $g\left(k, s^{\prime}\right)=f\left(y_{k}, s^{\prime}\right)$ for all $s^{\prime}>s$. We conclude that $z \neq \lim _{s} g(k, s)$ for all $k \in \omega$.

We will need a uniform version of the lemma above. For a set $A \subseteq \omega$, we define $\sup A=\max A$ if $A$ is finite and $\sup A=\infty$ otherwise.

Proposition 2.3. Suppose $\mathcal{S}=\left\{S_{n}\right\}_{n \in \omega}$ is uniformly $\Sigma_{2}^{0}$. Assume that there is a uniformly limitwise monotonic sequence $\mathcal{U}=\left\{U_{n}\right\}_{n \in \omega}$ such that $U_{n} \subseteq S_{n}$ and $\sup U_{n}=\sup S_{n} \in \omega \cup\{\infty\}$ for every $n \in \omega$. Then $\mathcal{S}$ is uniformly limitwise monotonic.

Proof. We carry out the proof of the lemma above uniformly in $n$. There is a computable function $h$ such that for all $z, n \in \omega$ we have

$$
z \in S_{n} \Longleftrightarrow W_{h(n, z)} \text { is finite. }
$$


Let $f(n, y, s)$ be a uniform limitwise monotonic approximation for the sequence $\mathcal{U}=\left\{U_{n}\right\}_{n \in \omega}$.

For each $n \in \omega$, let $\left\{\left(z_{k}^{n}, y_{k}^{n}, s_{k}^{n}\right)\right\}_{k \in \omega}$ be a uniformly computable listing of all triples such that $f\left(n, y_{k}^{n}, s_{k}^{n}\right) \geq z_{k}^{n}$. Define

$$
g(n, k, s)= \begin{cases}z_{k}^{n} & \text { if } W_{h\left(n, z_{k}^{n}\right), s} \subseteq W_{h\left(n, z_{k}^{n}\right), s_{k}^{n}} \\ f\left(n, y_{k}^{n}, s\right) & \text { otherwise. }\end{cases}
$$

Taking into account that $\sup U_{n}=\sup S_{n}$, one proves, as in the lemma above, that $\lambda n, k\left[\max _{s} g(n, k, s)\right]$ is total and $S_{n}=\operatorname{rng}\left(\lambda k\left[\max _{s} g(n, k, s)\right]\right)$ for $n \in \omega$.

\section{Proof of Theorem 1.3}

In order to prove Theorem 1.3 we need to build a sequence $\mathcal{S}=\left\{S_{n}\right\}_{n \in \omega}$ of infinite sets such that $\mathcal{S}$ satisfies the following conditions:

(a) $\mathcal{S}$ is not uniformly limitwise monotonic and

(b) $\mathcal{S}$ is uniformly limitwise monotonic relative to every hyperimmune degree.

The definition of the family $\mathcal{S}$ is simply a diagonalization construction. We define the $n$-th set $S_{n}$ as follows:

$$
S_{n}= \begin{cases}\omega-\left\{\max _{x} \varphi_{n}(x)\right\} & \text { if } \max _{x} \varphi_{n}(x) \text { exists } \\ \omega & \text { otherwise. }\end{cases}
$$

We assume for a contradiction that the sequence $\mathcal{S}$ is uniformly limitwise monotonic. Then there exists a computable function $g$ such that (1) for all $n, y \in \omega$ the value $\max _{x} g(n, y, x)$ exists and (2) $S_{n}=\operatorname{rng}\left(\lambda y\left[\max _{x} g(n, y, x)\right]\right)$ for all $n \in \omega$. Let $f$ be a computable function such that $\varphi_{f(n)}=\lambda x[g(n, 0, x)]$ for every $n$. Then $a_{n}=\max _{x} \varphi_{f(n)}(x)$ exists and so $a_{n} \notin S_{f(n)}$. On the other hand, we have $a_{n}=\max _{x} g(n, 0, x) \in S_{n}$ for every $n$. Hence, $\varphi_{f(n)} \neq \varphi_{n}$ for every $n \in \omega$. We have a contradiction with the Recursion Theorem. Thus, $\mathcal{S}$ is not uniformly limitwise monotonic.

Now we show that $\mathcal{S}$ is uniformly limitwise monotonic relative to every hyperimmune degree $\mathbf{x}$. Fix a function $r \leq_{T} \mathbf{x}$ such that no computable function dominates $r$. We define an $\mathbf{x}$-computable function $g$ as follows. First, set $g(n,\langle m, u\rangle, 0)=m$ for all $n, m$ and $u$. Supposing $g(n,\langle m, u\rangle, s)$ has been defined with value $k$, we set

$$
g(n,\langle m, u\rangle, s+1)= \begin{cases}k+1 & \text { if } u<s \text { and } \max _{x<t} \varphi_{n, t}(x)=k, \\ k & \text { otherwise, }\end{cases}
$$

where $t=\max \{r(k), s\}$. Fixing $n$ and $m$, one can see that $m \neq \max _{x} \varphi_{n}(x)$ if and only if $\max _{s} g(n,\langle m, u\rangle, s)=m$ for some $u$. Furthermore, if $k=\max _{s} g(n,\langle m, u\rangle, s)$ exists, then $k \neq \max _{x} \varphi_{n}(x)$.

Now assume that $\sup _{s} g(n,\langle m, u\rangle, s)=\infty$ for some $n, m$ and $u$. It must be the case that $\sup _{x} \varphi_{n}(x)=\infty$. For each $k \in \omega$, define $h(k)$ to be the least integer such that

$$
k<\max _{x<h(k)} \varphi_{n, h(k)}(x) .
$$

By our hypothesis, the computable function $h$ fails to dominate $r$. Therefore there must be an integer $k>m$ such that $h(k)<r(k)$. But the definition of $g$ ensures that $\max _{s} g(n,\langle m, u\rangle, s) \leq k$, contrary to our assumption. Indeed, suppose $g(n,\langle m, u\rangle, s)=k$ for some $s$. We show that $g(n,\langle m, u\rangle, s+1)=k$. Let $t=$ $\max \{r(k), s\}$. We have $t>h(k)$ and $\max _{x<t} \varphi_{n, t}(x) \geq \max _{x<h(k)} \varphi_{n, h(k)}(x)>k$. 
By the definition of $g, g(n,\langle m, u\rangle, s+1)=k$. This is a contradiction. Thus, $\max _{s} g(n, y, s)$ exists for every $n, y \in \omega$ and $S_{n}=\operatorname{rng}\left(\lambda y\left[\max _{s} g(n, y, s)\right]\right)$ for all $n \in \omega$. This proves the theorem.

\section{Proof of Theorem 1.4}

Recall that we have to build an infinite $\Sigma_{2}^{0}$ set $S$ which is not limitwise monotonic but is limitwise monotonic in every non-zero $\Delta_{2}^{0}$ degree. It is well-known that every non-zero $\Delta_{2}^{0}$ degree is hyperimmune.

Let $\left\{\psi_{n}\right\}_{n \in \omega}$ be a computable listing of all partial $\emptyset^{\prime}$-computable functions. We need to build a set $S \in \Sigma_{2}^{0}$ that satisfies the following requirements:

$N_{i}: \lambda y\left[\max _{x} \varphi_{i}(y, x)\right]$ is total and injective $\Longrightarrow S \neq \operatorname{rng}\left(\lambda y\left[\max _{x} \varphi_{i}(y, x)\right]\right)$;

$R_{n}: \psi_{n}$ is total and $\psi_{n}$ is not computably dominated $\Longrightarrow S$ is limitwise monotonic relative to $\psi_{n}$.

Note that by Proposition 2.1, the requirements $N_{i}$ guarantee that $S$ is not limitwise monotonic. To satisfy $R_{n}$ we define a (trace) function $\lambda m, s\left[g_{n}(m, s)\right]$ with the following properties:

1. The function $\lambda m, s\left[g_{n}(m, s)\right]$ is total and computable in $\psi_{n}$ if $\psi_{n}$ is total.

2. For each $n$ and $m$, the value of $g_{n}(m, s)$ is equal to $\langle n, m, k\rangle$ for some $k$.

3. The function $\lambda m\left[\max _{s} g_{n}(m, s)\right]$ is injective on its domain and satisfies the following sub-requirements for all $m \in \omega$ :

$R_{n, m}: \psi_{n}$ is total and $\psi_{n}$ is not computably dominated $\Longrightarrow \max _{s} g_{n}(m, s)$ exists, and $\max _{s} g_{n}(m, s) \in S$.

Note that, by Proposition 2.3 , if the requirements $R_{n, m}$ are met for every $m$, then the requirement $R_{n}$ is met. We order the requirements effectively in such a way that $N_{i}$ is of a higher priority than $R_{n, m}$ if $i \leq\langle n, m\rangle$.

The strategy for $N_{i}$ is to keep $\max _{x} \varphi_{i}(j, x)$ for at least one $j$ outside $S$, where $j \leq i$. All these values are restrained for the $R_{n, m}$-requirements of lower priority. The strategy can be injured by at most $i$ many traces $\max _{s} g_{n}(m, s) \in S$ that are of higher priority than $N_{i}$ (that is, $\langle m, n\rangle<i$ ). Thus, $N_{i}$ wins by keeping $\max _{x} \varphi_{i}(j, x)$ outside of $S$ for some $j \leq i$.

The strategy for each $R_{n, m}$ is to define traces $g_{n}(m, s)$ for $s \in \omega$, avoiding the numbers restrained by $N_{i}$-requirements of higher priorities. The definition will be similar to the one we had in the proof of Theorem 1.3. We need to keep in $S$ the value of $\max _{s} g_{n}(m, s)$. Here we have to be more careful because it may happen that $\psi_{n}$ is not total. In this case the naive definition of $S$ (e.g., as the collection of final traces for all $n$ and $m$ ) can cause $S \notin \Sigma_{2}^{0}$. To circumvent this problem we give a more accurate definition of $S$ (see below).

For each $n \in \omega$, define a partial function $g_{n}$ by induction as follows. Set $g_{n}(m, 0)=\langle n, m, 0\rangle$ and

$$
g_{n}(m, s+1)= \begin{cases}\langle n, m, k+1\rangle \quad & \text { if } g_{n}(m, s) \downarrow=\langle n, m, k\rangle, \quad \psi_{n}(k) \downarrow, \text { and } \\ & \langle n, m, k\rangle \in\left\{\max _{x<t} \varphi_{i, t}(j, x) \mid j \leq i \leq\langle n, m\rangle\right\}, \\ & \text { where } t=\max \left\{\psi_{n}(k), s\right\}, \\ \langle n, m, k\rangle \quad & \text { if } g_{n}(m, s) \downarrow=\langle n, m, k\rangle, \psi_{n}(k) \downarrow, \text { and } \\ & \langle n, m, k\rangle \notin\left\{\max _{x<t} \varphi_{i, t}(j, x) \mid j \leq i \leq\langle n, m\rangle\right\}, \\ & \text { where } t=\max \left\{\psi_{n}(k), s\right\}, \\ & \text { otherwise. }\end{cases}
$$


By its definition, $\left\{g_{n}\right\}_{n \in \omega}$ is a uniformly computable sequence of partial $\emptyset^{\prime}$ computable functions. Now set

$$
\begin{aligned}
S=\{ & \langle n, m, k\rangle \mid(\exists s)\left[g_{n}(m, s)=\langle n, m, k\rangle \&\right. \\
& \left.\left.(\forall u \geq s)(\forall i \leq\langle n, m\rangle)(\forall j \leq i)\left[\langle n, m, k\rangle \neq \max _{x<u} \varphi_{i, u}(j, x)\right]\right]\right\} .
\end{aligned}
$$

By its definition, $S$ is $\Sigma_{2}^{0}$. Furthermore, if $\langle n, m, k\rangle \in S$, then

$$
\langle n, m, k\rangle=\max _{s} g_{n}(m, s) \text {. }
$$

Indeed, let $\langle n, m, k\rangle \in S$. Then $g_{n}(m, s)=\langle n, m, k\rangle$ and

$$
(\forall u \geq s)(\forall i \leq\langle n, m\rangle)(\forall j \leq i)\left[\langle n, m, k\rangle \neq \max _{x<u} \varphi_{i, u}(j, x)\right],
$$

for some $s$. It follows that $g_{n}(m, u)=\langle n, m, k\rangle$ or $g_{n}(m, u) \uparrow$ for all $u \geq s$. Thus, for every $n, m$ there exists at most one $k$ such that $\langle n, m, k\rangle \in S$.

First, we show that $S$ is limitwise monotonic relative to every $A \in \Delta_{2}^{0}-\Delta_{1}^{0}$. Fixing $A \in \Delta_{2}^{0}-\Delta_{1}^{0}$, since $A$ is hyperimmune, there is an $n \in \omega$ such that $\psi_{n}$ is total, $\psi_{n} \leq_{T} A$, and $\psi_{n}$ is not dominated by any computable function. Thus, $g_{n}$ is a total function and $g_{n} \leq_{T} A$.

We claim that if $\max _{s} g_{n}(m, s)=\langle n, m, k\rangle$, then $\langle n, m, k\rangle \in S$. Fix an $s>\psi_{n}(k)$ such that $g_{n}(m, u)=\langle n, m, k\rangle$ for all $u \geq s$. Then we have

$$
(\forall u \geq s)(\forall i \leq\langle n, m\rangle)(\forall j \leq i)\left[\langle n, m, k\rangle \neq \max _{x<u} \varphi_{i, u}(j, x)\right] .
$$

Therefore, $\langle n, m, k\rangle \in S$.

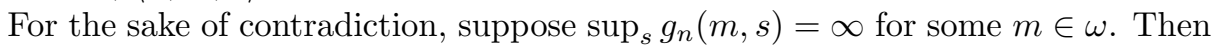
the finite set $I=\left\{\langle i, j\rangle \mid j \leq i \leq\langle n, m\rangle \& \sup _{x} \varphi_{i}(j, x)=\infty\right\}$ is not empty. Choose an integer $s_{0} \in \omega$ such that

$$
\text { if } j \leq i \leq\langle n, m\rangle \text { and }\langle i, j\rangle \notin I \text {, then } \max _{x} \varphi_{i}(j, x)<s_{0} \text {. }
$$

For every $k \in \omega$, define $h(k)$ to be the least integer such that

$$
\langle n, m, k\rangle<\min _{\langle i, j\rangle \in I} \max _{x<h(k)} \varphi_{i, h(k)}(j, x) .
$$

By the choice of $n$, there must be an integer $k>s_{0}$ such that $h(k)<\psi_{n}(k)$.

The definition of $g_{n}$ ensures that $\max _{s} g_{n}(m, s) \leq\langle n, m, k\rangle$, contrary to our assumption. Indeed, assume $g_{n}(m, s)=\langle n, m, k\rangle$ for some $s$. We show that $g_{n}(m, s+1)=\langle n, m, k\rangle$.

Let $t=\max \left\{\psi_{n}(k), s\right\}$. Note that $t \geq \psi_{n}(k)>h(k)$. If $j \leq i \leq\langle n, m\rangle$ and $\langle i, j\rangle \in I$, then

$$
\max _{x<t} \varphi_{i, t}(j, x) \geq \max _{x<h(k)} \varphi_{i, h(k)}(j, x)>\langle n, m, k\rangle .
$$

If $j \leq i \leq\langle n, m\rangle$ and $\langle i, j\rangle \notin I$, then

$$
\langle n, m, k\rangle \geq k>s_{0}>\max _{x} \varphi_{i}(j, x) \geq \max _{x<t} \varphi_{i, t}(j, x) .
$$

Thus, whether $\langle i, j\rangle \in I$ or $\langle i, j\rangle \notin I$,

$$
\langle n, m, k\rangle \notin\left\{\max _{x<t} \varphi_{i, t}(j, x) \mid j \leq i \leq\langle n, m\rangle\right\} .
$$

By the definition of $g_{n}$, we have $g_{n}(m, s+1)=\langle n, m, k\rangle$.

Thus, $\max _{s} g_{n}(m, s)$ exists for every $m \in \omega, \lambda m\left[\max _{s} g_{n}(m, s)\right]$ is injective, and $\operatorname{rng}\left(\lambda m\left[\max _{s} g_{n}(m, s)\right]\right) \subseteq S$. By Lemma 2.2, the infinite set $S$ is limitwise monotonic in $A$. 
We prove that $S$ is not limitwise monotonic. Suppose $S$ is limitwise monotonic. Then, by Proposition 2.1, there is an $i \in \omega$ such that

$$
S=\operatorname{rng}\left(\lambda j\left[\max _{x} \varphi_{i}(j, x)\right]\right),
$$

where $\lambda j\left[\max _{x} \varphi_{i}(j, x)\right]$ is total and injective. Hence, the finite set

$$
\left\{\max _{x} \varphi_{i}(j, x) \mid j \leq i\right\} \subseteq S
$$

has cardinality $i+1$. Therefore, there are integers $n, m, k$ and $j \leq i$ such that $i \leq\langle n, m\rangle$ and $\max _{x} \varphi_{i}(j, x)=\langle n, m, k\rangle \in S$ (since for every $n$ and $m$ there exists at most one $k$ such that $\langle n, m, k\rangle \in S$ ). But by the definition of $S$ we have $\langle n, m, k\rangle \neq \max _{x} \varphi_{i}(j, x)$ for $j \leq i \leq\langle n, m\rangle$. Thus, $S$ is not limitwise monotonic.

\section{Proof of Theorem 1.6}

Recall that a tree $T \subseteq 2^{<\omega}$ is splitting if for every $\sigma \in T$ there exist incomparable strings $\rho_{0}, \rho_{1} \in T$ such that $\sigma \subseteq \rho_{0}$ and $\sigma \subseteq \rho_{1}$. Theorem 1.6 follows from the technical lemma below:

Lemma 5.1. Suppose a sequence $\left\{S_{n}\right\}_{n \in \omega}$ is not uniformly limitwise monotonic. Let $\Phi$ be a Turing operator and $T \subseteq 2^{<\omega}$ be a non-empty computable splitting tree. Then there is a non-empty computable splitting subtree $T_{1} \subseteq T$ such that the condition

$$
(\forall n)(\forall k)\left[\max _{s} \Phi^{X}(n, k, s) \text { exists }\right] \&(\forall n)\left[S_{n}=\operatorname{rng}\left(\lambda k\left[\max _{s} \Phi^{X}(n, k, s)\right]\right)\right]
$$

(that is, $\Phi^{X}$ is a uniform limitwise monotonic approximation of $\left\{S_{n}\right\}_{n \in \omega}$ ) fails for every infinite path $X$ through $T_{1}$.

First we prove the theorem using Lemma [5.1] and then we prove the lemma.

Proof of Theorem 1.6. Let $\left\{S_{n}\right\}_{n \in \omega}$ be uniformly limitwise monotonic in all degrees except perhaps countably many. We prove that $\left\{S_{n}\right\}_{n \in \omega}$ has to be uniformly limitwise monotonic.

Towards a contradiction, assume that $\left\{S_{n}\right\}_{n \in \omega}$ is not uniformly limitwise monotonic. We will build uncountably many sets $X$ satisfying the requirements

$$
P_{m}^{X}: \Phi_{m}^{X} \text { is not a uniform limitwise monotonic approximation of }\left\{S_{n}\right\}_{n \in \omega}
$$

for a fixed effective list of all Turing operators $\left\{\Phi_{m}\right\}_{m \in \omega}$.

To make sure that each requirement $P_{m}^{X}$ is met for $X$, we apply Lemma 5.1 , More specifically, for each computable splitting tree $T$ and each Turing operator $\Phi$, we fix a subtree $P(\Phi, T) \subseteq T$ such that $\Phi^{X}$ is not a uniform limitwise monotonic approximation of $\left\{S_{n}\right\}_{n \in \omega}$ for every infinite path $X$ through $P(\Phi, T)$.

For a non-empty computable splitting tree $T \subseteq 2^{<\omega}$, define

$$
R_{0}(T)=\left\{\sigma \in T \mid \sigma \subseteq \rho_{0} \text { or } \rho_{0} \subseteq \sigma\right\}
$$

and

$$
R_{1}(T)=\left\{\sigma \in T \mid \sigma \subseteq \rho_{1} \text { or } \rho_{1} \subseteq \sigma\right\},
$$

where $\rho_{0}, \rho_{1} \in T$ are incomparable. We have $R_{0}(T) \subseteq T, R_{1}(T) \subseteq T,\left[R_{0}(T)\right] \cap$ $\left[R_{1}(T)\right]=\emptyset$, and $R_{0}(T)$ and $R_{1}(T)$ are non-empty (computable) splitting trees. 
Let $h: \omega \longrightarrow\{0,1\}$ be any (not necessarily computable) function. Let $X_{h}$ be an infinite path through $\bigcap_{k \in \omega} T_{k}$, where $\left\{T_{k}\right\}_{k \in \omega}$ is the family of computable splitting trees defined recursively as follows:

(1) $T_{0}=2^{<\omega}$

(2) $T_{2 m+1}=R_{h(m)}\left(T_{2 m}\right), m \in \omega$;

(3) $T_{2 m+2}=P\left(\Phi_{m}, T_{2 m+1}\right), m \in \omega$.

Then the set $X_{h}$ satisfies $P_{m}$ for all $m \in \omega$, and the map $h \mapsto X_{h}$ is injective. Thus, $\left\{S_{n}\right\}_{n \in \omega}$ is not uniformly limitwise monotonic relative to $2^{\aleph_{0}}$ many different oracles. This is a contradiction.

Proof of Lemma 5.1. For each $n$ consider the set

$$
\begin{aligned}
& M_{n}=\left\{y \mid(\exists k)(\exists s)(\exists \sigma \in T)\left[\Phi^{\sigma}(n, k, s) \downarrow\right.\right.=y \& \\
&\left.\left.(\forall \tau \in T)\left(\forall s^{\prime}\right)\left(\forall y^{\prime}\right)\left[\sigma \subseteq \tau \& \Phi^{\tau}\left(n, k, s^{\prime}\right) \downarrow=y^{\prime} \rightarrow y^{\prime} \leq y\right]\right]\right\} .
\end{aligned}
$$

We have the following cases:

Case 1. There exists $y \in M_{n}-S_{n}$ for some $y, n$.

Then we can choose $\sigma \in T$ such that $y=\Phi^{\sigma}(n, k, s) \downarrow$ and

$$
\left.(\forall \tau \in T)\left(\forall s^{\prime}\right)\left(\forall y^{\prime}\right)\left[\sigma \subseteq \tau \& \Phi^{\tau}\left(n, k, s^{\prime}\right) \downarrow=y^{\prime} \rightarrow y^{\prime} \leq y\right]\right\}
$$

for some $k, s$. We can set $T_{1}=\{\tau \in T \mid \tau \subseteq \sigma$ or $\sigma \subseteq \tau\}$, since

$$
y=\max _{s} \Phi^{X}(n, k, s) \notin S_{n}
$$

for every infinite path $X$ through $T_{1}$.

Case 2. There exists $y \in S_{n}-M_{n}$ for some $y, n$.

Define a computable function

$$
f: 2^{<\omega} \longrightarrow T
$$

by recursion as follows:

(1) Define $f(\lambda)=\lambda$, where $\lambda$ is the empty string.

(2) Suppose we have $f(\alpha)=\sigma$ and the length of $\alpha$ has the form $\ell=\langle k, t\rangle$ (it is important for us to make sure that for each $k$ we will have infinitely many corresponding lengths of the form $\ell=\langle k, i\rangle)$. Suppose $\Phi^{\sigma}(n, k, s)[t] \downarrow=y$ for some $s<t$. Since $y \notin M_{n}$ we can find a string $\tau \in T, \sigma \subseteq \tau$, such that $\Phi^{\tau}\left(n, k, s^{\prime}\right) \downarrow>y$ for some $s^{\prime}$. If $\Phi^{\sigma}(n, k, s)[t] \uparrow$ or $\Phi^{\sigma}(n, k, s)[t] \downarrow \neq y$ for every $s<t$, then set $\tau=\sigma$. Then we choose incomparable strings $\rho_{0}, \rho_{1} \in T$, such that $\tau \subseteq \rho_{0}$ and $\tau \subseteq \rho_{1}$, and define $f(\alpha * i)=\rho_{i}$ for $i \in\{0,1\}$.

Let $T_{1}=\left\{\sigma \mid\left(\exists \beta \in 2^{<\omega}\right)[\sigma \subseteq f(\beta)]\right\}$. Suppose $X$ is an infinite path through $T_{1}$. For every $k \in \omega$ we have only two possibilities. First, there is a string $\tau \subseteq X$ such that $\Phi^{\tau}(n, k, s) \downarrow>y$ for some $s$. Second, there are no $\sigma \in T_{1}$ and $s$ such that $\Phi^{\sigma}(n, k, s) \downarrow=y$. Hence,

$$
y \in S_{n}-\operatorname{rng}\left(\lambda k\left[\max _{s} \Phi^{X}(n, k, s)\right]\right)
$$

for every infinite path $X$ through $T_{1}$.

Case 3. $S_{n}=M_{n}$ for every $n$.

In this case, the sequence $\left\{S_{n}\right\}_{n \in \omega}$ is uniformly $\Sigma_{2}^{0}$ (see the definition of $M_{n}$ ). 
For every $m \in \omega$, let $\psi(n, m)=\left\langle\sigma_{n, m}, k_{n, m}, y_{n, m}\right\rangle$ be the first found triple $\langle\sigma, k, y\rangle$ such that $\sigma \in T$ and $\Phi^{\sigma}(n, k, s) \downarrow=y \geq m$ for some $s$. Set $\psi(n, m)$ to be undefined if such a triple does not exist. Clearly, $\psi$ is a partial computable function.

Note that if $\psi(n, m)$ is defined, then $\psi\left(n, m^{\prime}\right)$ is defined for every $m^{\prime}<m$.

Suppose first that $\psi(n, m)$ is undefined for some $m \in S_{n}$. We have

$$
m \in S_{n}-\operatorname{rng}\left(\lambda k, s\left[\Phi^{X}(n, k, s)\right]\right) \subseteq S_{n}-\operatorname{rng}\left(\lambda k\left[\max _{s} \Phi^{X}(n, k, s)\right]\right)
$$

for every infinite path $X$ through $T$. Then we can set $T_{1}=T$. Hence we assume that $\psi(n, m)$ is defined for every $n, m \in \omega$ such that $m \leq \sup S_{n}$.

The intuition behind the formal argument below is as follows. We attempt to define a uniform limitwise monotonic approximation of some subset of $\left\{S_{n}\right\}_{n \in \omega}$ using the fact that $\left\{S_{n}\right\}_{n \in \omega}=\left\{M_{n}\right\}_{n \in \omega}$. Since by Proposition 2.3 we cannot succeed, we will have to have an infinite splitting subtree of $T$ witnessing this failure.

Define partial computable functions

$$
f: \omega \times \omega \times 2^{<\omega} \longrightarrow T \text { and } g: \omega \times \omega \times 2^{<\omega} \longrightarrow \omega
$$

by recursion as follows:

(1) With $\lambda$ the empty string, define

$$
f(n, m, \lambda)=\sigma_{n, m} \text { and } g(n, m, \lambda)=y_{n, m}
$$

if $\psi(n, m) \downarrow=\left\langle\sigma_{n, m}, k_{n, m}, y_{n, m}\right\rangle$ and set $f(n, m, \lambda) \uparrow$ and $g(n, m, \lambda) \uparrow$ if $\psi(n, m)$ is undefined. Note that $f(n, m, \lambda)$ and $g(n, m, \lambda)$ are defined if $m \leq \sup S_{n}$

(2) Suppose $f(n, m, \alpha)$ and $g(n, m, \alpha)$ have already been defined. Let $\langle\tau, y\rangle$ be the first found pair such that $\tau \in T, f(n, m, \alpha) \subseteq \tau, g(n, m, \alpha)<y$, and $\Phi^{\tau}\left(n, k_{n, m}, s\right) \downarrow=y$ for some $s$. Then we choose incomparable strings $\rho_{0}, \rho_{1} \in T$ such that $\tau \subseteq \rho_{0}, \tau \subseteq \rho_{1}$. We set

$$
f(n, m, \alpha * i)=\rho_{i} \text { and } g(n, m, \alpha * i)=y
$$

for $i \in\{0,1\}$. If $f(n, m, \alpha), g(n, m, \alpha)$ are undefined or $\langle\tau, y\rangle$ does not exist, then $f(n, m, \alpha * i)$ and $g(n, m, \alpha * i)$ remain undefined.

It is crucial that if $g(n, m, \alpha)$ is defined and $g(n, m, \alpha * i)$ is undefined, then $g(n, m, \alpha) \in M_{n}=S_{n}$.

Suppose that for every $n, m \in \omega$ there is a string $\beta \in 2^{<\omega}$ such that $g(n, m, \beta)$ is undefined. By our assumption, $\beta$ is not the empty string if $m \leq \sup S_{n}$. Hence, if $m \leq \sup S_{n}$, then there is a string $\alpha \in 2^{<\omega}$ such that $g(n, m, \alpha)$ is defined but $g(n, m, \alpha * 0)$ is undefined (recall that $g(n, m, \alpha * 0) \downarrow$ iff $g(n, m, \alpha * 1) \downarrow)$.

Consider the sequence $\left\{U_{n}\right\}_{n \in \omega}$, where $U_{n}=\{h(n, m) \mid g(n, m, \lambda) \downarrow\}$, and $h(n, m)=\min \left\{y \mid\left(\exists \alpha \in 2^{<\omega}\right)[g(n, m, \alpha) \downarrow=y\right.$ and $\left.g(n, m, \alpha * 0) \uparrow]\right\}$.

We show that $\left\{U_{n}\right\}_{n \in \omega}$ is uniformly limitwise monotonic. By the definition of $h$, $\operatorname{dom}(h)=\operatorname{dom}(\psi)$ is c.e., and $g(n, m, \alpha * i) \downarrow$ implies $g(n, m, \alpha)<g(n, m, \alpha * i)$ for each $i \in\{0,1\}$. Thus, given $m$ and $n$, we can monotonically and uniformly in $n$ and $m$ approximate $h(n, m)$ as follows. Let $g_{s}$ be the (finite) part of $g$ computed at stage $s$. As usual, we may assume that $g_{s}(n, m, \alpha) \downarrow$ implies that the length of $\alpha$ is less than $s$. Therefore, given $n, m, u, s \in \omega$ such that $g_{u}(n, m, \lambda) \downarrow$ and $s \geq u$, we can effectively choose $k(n, m, u, s)$ least such that

$$
g_{s}(n, m, \alpha) \downarrow=k(n, m, u, s) \text { and } g_{s}(n, m, \alpha * i) \uparrow
$$


for some $i \in\{0,1\}$ and $\alpha \in 2^{<\omega}$. Suppose $g_{s}(n, m, \alpha) \uparrow, g_{u}(n, m, \lambda) \downarrow$ and $s \geq u$. Let $\gamma \subset \alpha$ be the $\subseteq$-maximal substring of $\alpha$ such that $g_{s}(n, m, \gamma) \downarrow$. Such a $\gamma$ exists because $g_{s}(n, m, \lambda) \downarrow=g_{u}(n, m, \lambda)$. Fix $i \in\{0,1\}$ so that $\gamma * i \subseteq \alpha$. By the choice of $\gamma$, we have $g_{s}(n, m, \gamma) \downarrow, g_{s}(n, m, \gamma * i) \uparrow$, and, therefore,

$$
g(n, m, \alpha) \downarrow \Longrightarrow g(n, m, \alpha) \geq g(n, m, \gamma) \geq k(n, m, u, s) .
$$

Thus, $k(n, m, u, s+1) \geq k(n, m, u, s)$ for every $n, m, u, s \in \omega$ such that $g_{u}(n, m, \lambda) \downarrow$ and $s \geq u$. Furthermore, for every $\alpha$ we have $g(n, m, \alpha * 0) \downarrow$ if and only if $g(n, m, \alpha * 1) \downarrow$. This implies $\max _{s \geq u} k(n, m, u, s)=h(n, m)$. It remains to define a total limitwise monotonic approximation of $h(n, m)$ using the partial approximation $k(n, m, u, s)$, as follows. Let $M(n, z)$ be a total computable function such that

$$
h(n, m) \downarrow \Longleftrightarrow g(n, m, \lambda) \downarrow \Longleftrightarrow \psi(n, m) \downarrow \Longleftrightarrow(\exists z)[M(n, z)=m] .
$$

Define $u(n, z)$ to be equal to the least stage $u$ such that $g_{u}(n, M(n, z), \lambda) \downarrow$. Set

$$
H(n, z, s)=k(n, M(n, z), u(n, z), s+u(n, z)) .
$$

The function $H(n, z, s)$ is total and non-decreasing in $s$. Furthermore,

$$
\max _{s} H(n, z, s)=\max _{s} k(n, M(n, z), u(n, z), s+u(n, z))=h(n, M(n, z))
$$

for every $n$ and $z$. Hence, $H$ is a uniform limitwise monotonic approximation of $\left\{U_{n}\right\}_{n \in \omega}$. We have $\sup S_{n}=\sup U_{n}, \operatorname{since} h(n, m) \downarrow \geq m$ for every $m \leq \sup S_{n}$ and $U_{n} \subseteq M_{n}=S_{n}$. By Proposition 2.3. $\left\{S_{n}\right\}_{n \in \omega}$ is uniformly limitwise monotonic, contrary to the hypothesis.

Thus, there exist $n, m \in \omega$ such that $f(n, m, \beta)$ and $g(n, m, \beta)$ are defined for every $\beta \in 2^{<\omega}$. Set

$$
T_{1}=\left\{\sigma \mid\left(\exists \beta \in 2^{<\omega}\right)[\sigma \subseteq f(n, m, \beta)]\right\} \subseteq T .
$$

We have $\lim _{\alpha: f(n, m, \alpha) \subset X} g(n, m, \alpha)=\infty$ for every infinite path $X$ through $T_{1}$ and $\Phi^{f(n, m, \alpha)}\left(n, k_{n, m}, s_{\alpha}\right)=g(n, m, \alpha)$ for some $s_{\alpha}$. Therefore $\max _{s} \Phi^{X}\left(n, k_{n, m}, s\right)$ does not exist for every infinite path $X$ through $T_{1}$.

\section{Applichtions}

Our goal is to to apply the results obtained in the previous sections to study degree spectra of structures in the classes of abelian groups, equivalence structures, and models of $\aleph_{1}$-categorical theories. For a background on the general theory of computable structures, see [8].

6.1. Abelian groups. Let $p_{0}, p_{1}, \ldots$ be the sequence of prime numbers listed in increasing order. For a prime $p$ and integer $n$, the cyclic group of order $p^{n}$ is denoted by $Z_{p^{n}}$. For an infinite set $S(0 \notin S)$ and prime $p$ we denote by $A_{p}(S)$ the group $A_{p}(S)=\bigoplus_{n \in S} Z_{p^{n}}$. We need the following uniform version of a well-known result of Khisamiev ([18], Theorem 3.4):

Lemma 6.1. A sequence $\mathcal{S}=\left\{S_{n}\right\}_{n \in \omega}$ of infinite sets of positive integers is uniformly limitwise monotonic if and only if the abelian group $G=\bigoplus_{n \in \omega} A_{p_{n}}\left(S_{n}\right)$ has a computable copy.

Proof. Assume $G=\bigcup_{s \in \omega} G_{s}$ is computable, where $G_{s}$ is the part of $G$ enumerated at stage $s$. By the definition of $G$, for every element $a$ of $G$ we can effectively choose a positive integer $m_{a}$ least such that $m_{a} a=0$. We have $a \in A_{p_{n}}\left(S_{n}\right)$ if and only if $m_{a}$ is a power of $p_{n}$. Therefore, given $n \in \omega$ and a computable index 
for $G$, we can uniformly compute an index for the computable subgroup $A_{p_{n}}\left(S_{n}\right)$ of $G$. Also, given an index for $A_{p_{n}}\left(S_{n}\right)$, we can uniformly pass to a computable index for $C_{n}=\left\{c \in A_{p_{n}}\left(S_{n}\right) \mid p_{n} c=0\right\}=\left\{c_{i}\right\}_{i \in \omega}$. Let $f(n, i, s)=\max \{h \mid$ $\left.h=1 \vee\left(\exists b \in G_{s}\right)\left[(\forall k<h) p_{n}^{k} b \neq 0 \wedge p_{n}^{h-1} b=c_{i}\right]\right\}$. By the definition of $C_{n}$, we have $S_{n}=\operatorname{rng}\left(\lambda i\left[\max _{s} f(n, i, s)\right]\right)$. The function $f(n, i, s)$ is a uniform limitwise monotonic approximation of $\mathcal{S}$.

Now suppose $\mathcal{S}=\left\{S_{n}\right\}_{n \in \omega}$ is uniformly limitwise monotonic. By Proposition 2.1, we can choose a uniform limitwise monotonic approximation $f(n, i, s)$ of $\mathcal{S}$ such that $\lambda i\left[\max _{s} f(n, i, s)\right]$ is injective for every $n$. We have $G=\bigcup_{t \in \omega} \bigoplus_{n \leq t}\left(\bigoplus_{i \leq t} Z_{p_{n}^{h(n, i, t)}}\right)$, where $h(n, i, t)=\max _{s \leq t} f(n, i, s)$ and $\bigoplus_{n \leq t}\left(\bigoplus_{i \leq t} Z_{p_{n}^{h(n, i, t)}}\right)$ is a naturally defined subgroup of the group $\bigoplus_{n \leq t+1}\left(\bigoplus_{i \leq t+1} Z_{p_{n}^{h(n, i, t+1)}}\right)$.

Proof of Theorem 1.7. The first part of the theorem follows from Lemma 6.1 (relativized) and Theorem 1.3. and the second part of the theorem follows from Theorem 3.4 (relativized) of [18] and Theorem 1.4.

Proof of Theorem 1.8. The theorem follows from Lemma 6.1 (relativized) and Theorem 1.6 .

6.2. Equivalence relations. To apply our computably-theoretic results we need the following observation (see, e.g., [5] or [2] for a proof):

Lemma $6.2([2)$. Suppose $\Theta$ is an equivalence structure in which all equivalence classes are finite and have distinct cardinalities $c_{0}, c_{1}, \ldots$ Then $\Theta$ has a computable copy if and only if $C=\left\{c_{0}, c_{1}, \ldots\right\}$ is limitwise monotonic.

Now the proof of the following theorem follows from Lemma 6.2 and Theorem 1.4

Theorem 6.3. There exists an equivalence structure $\Theta$ such that $\operatorname{Deg} S p(\Theta)$ contains a $\Delta_{2}^{0}$ degree $\mathbf{a}$ if and only if $\mathbf{a}>0$.

6.3. $\aleph_{1}$-categorical theories. Recall that a first order complete theory $T$ is $\aleph_{1}$ categorical if all models of $T$ of cardinality $\aleph_{1}$ are isomorphic. There are many natural examples of $\aleph_{1}$-categorical theories: the theory of algebraically closed fields of a given characteristic, the theory of vector spaces over a given countable field, and the theory of one successor function on the natural numbers. Baldwin and Lachlan [1] showed that all models of a given $\aleph_{1}$-categorical theory $T$ with more than one countable model (up to isomorphism) form an elementary chain $\mathcal{A}_{0} \preceq$ $\mathcal{A}_{1} \preceq \ldots \preceq \mathcal{A}_{\omega}$ of length $\omega+1$, where $\preceq$ stands for an elementary embedding. In this chain $\mathcal{A}_{0}$ is the prime model of $T$ and $\mathcal{A}_{\omega}$ is the saturated model of $T$. A natural question arises for a given $\aleph_{1}$-categorical theory $T$ : Which models in the corresponding elementary chain are computable? This is known as the spectra problem for $\aleph_{1}$-categorical theories [19]. Goncharov [7] asked whether the prime model $\mathcal{A}_{0}$ of $T$ is computable, given that one of the models $\mathcal{A}_{i}$ in the elementary chain is computable.

The problem of Goncharov was resolved in [19]. Recall that a model $\mathcal{M}$ of a theory $T$ is minimal if there is no formula $\phi(x)$ such that the sets $\{m \mid \mathcal{M}=\phi(m)\}$ and $\{m \mid \mathcal{M} \models \neg \phi(m)\}$ are infinite. A theory $T$ is strongly minimal if all models of $T$ are minimal. A theory is algebraically trivial if the algebraic closure of every set $X$ in every model of the theory equals the union of the algebraic closures of elements of $X$. 
Theorem 6.4 ([19]). For every given set $S$ there exists an $\aleph_{1}$-categorical but not $\aleph_{0}$-categorical theory $T_{S}$ with the following properties:

(1) The theory $T_{S}$ is strongly minimal and algebraically trivial.

(2) Every (countable) non-prime model of $T_{S}$ has a computable copy if and only if $S \in \Sigma_{2}^{0}$.

(3) The prime model of $T_{S}$ has a computable copy if and only if $S$ is limitwise monotonic.

Theorem 6.4 and the existence of a $\Sigma_{2}^{0}$ set which is not limitwise monotonic (see, e.g., [19] or Proposition 3.8 of [18]) implies that there is a strongly minimal and algebraically trivial $\aleph_{1}$-categorical theory such that every (countable) non-prime model of $T$ is computable, but the prime model of $T$ is not computable. This is a negative solution to Goncharov's problem in a strong form. However, Theorem 1.4 combined with Theorem 6.4 has an even stronger consequence:

Theorem 6.5. There exists an $\aleph_{1}$-categorical but not $\aleph_{0}$-categorical theory $T$ with the following properties:

(1) The theory $T$ is strongly minimal and algebraically trivial.

(2) Each (countable) non-prime model of T has a computable copy.

(3) The degree spectrum DegSp $\left(\mathcal{A}_{0}\right)$ of the prime model of $T$ contains a $\Delta_{2}^{0}$ degree $\mathbf{a}$ if and only if $\mathbf{a}>0$.

\section{REFERENCES}

1. Baldwin, J.T. and Lachlan, A.H. On strongly minimal sets. J. Symbolic Logic, 36, 1971, pp. 79-96. MR0286642 (44:3851)

2. Calvert, W., Cenzer, D., Harizanov, V., and Morozov, A. Effective categoricity of equivalence structures. Annals of Pure and Applied Logic, 141, 2006, pp. 61-78. MR2229930|(2007j:03048)

3. Csima, B., Hirschfeldt, D., Knight, J., and Soare, R. Bounding prime models. J. Symbolic Logic, 69, Issue 4, 2004, pp. 1117-1142. MR2135658 (2005m:03065)

4. Csima, B. and Kalimullin, I. Degree spectra and immunity properties. Math. Log. Q., 56(1), 2010, pp. 67-77. MR2598838 (2011i:03038)

5. Cenzer, D., Harizanov, V., and Remmel, J. $\Sigma_{1}^{0}$ and $\Pi_{1}^{0}$ equivalence structures. Mathematical Theory and Computational Practice, K. Ambos-Spies, B. Loewe, and W. Merkle, eds., Lect. Notes in Comput. Sci., 5635, Springer, 2009, pp. 99-108. MR2545884 (2011h:03054)

6. Downey, R., Kach, A., and Turetsky, D. Limitwise monotonic functions and their applications. Proceedings of the Eleventh Annual Asian Logic Conference, World Sci. Publ., Hackensack, NJ, 2012, pp. 59-85. MR2868506 (2012m:03084)

7. Ershov, Yu. and Goncharov, S. (editors). Logic Notebook. Open questions in logic. Novosibirsk University Press, 1989.

8. Ershov, Yu. and Goncharov, S. Constructive models. Siberian School of Algebra and Logic, Consultants Bureau, New York, 2000. MR1749622 (2002a:03069)

9. Frolov, A., Harizanov, V., Miller, R., Kalimullin, I., and Kudinov, O. Degree spectra of $\operatorname{high}_{n}$ and nonlown degrees. Journal of Logic and Computation, 22, 2012, pp. 755-777, doi:10.1093/logcom/exq041

10. Goncharov, S., Harizanov, V., Knight, J., McCoy, C., Miller, R., and Solomon, R. Enumerations in computable structure theory. Annals of Pure and Applied Logic, 136, 2005, pp. 219-246. MR2169684 (2006f:03071)

11. Harris, K. $\eta$-representation of sets and degrees. J. Symbolic Logic, 73(4), 2008, pp. 1097-1121. MR2467206 (2009k:03066)

12. Hirschfeldt, D. Prime models of theories of computable linear orderings. Proceedings of the American Mathematical Society, Vol. 129, No. 10, 2001, pp. 3079-3083. MR.1840114 (2002d:03062)

13. Hirschfeldt, D., Miller, R., and Podzorov, S. Order-computable sets. Notre Dame J. Formal Logic, Volume 48, Number 3, 2007, pp. 317-347. MR2336351 (2008g:03075) 
14. Hirschfeldt, D., Khoussainov, B., and Semukhin P. An uncountably categorical theory whose only computably presentable model is saturated. Notre Dame J. Formal Logic, 47, 2006, No. 1, pp. 63-71. MR2211182 (2007b:03047)

15. Hirschfeldt, D., Khoussainov, B., Shore, R., and Slinko, A. Degree spectra and computable dimensions in algebraic structures. Annals of Pure and Applied Logic, 115, 2002, pp. 71-113. MR.1897023 (2003d:03060)

16. Kach, A. Computable shuffle sums of ordinals. Archive for Mathematical Logic, 47(3), 2008, pp. 211-219. MR2415492(2009e:03078)

17. Kach, A. and Turetsky, D. Limitwise monotonic functions, sets, and degrees on computable domains. J. Symbolic Logic, 75:1, 2010, 131-154. MR2605885 (2011k:03093)

18. Khisamiev, N. Constructive abelian groups. In: Handbook of Recursive Mathematics, Vol. 2, volume 139 of Stud. Logic Found. Math., North-Holland, Amsterdam, 1998, pp. 1177-1231. MR.1673602 (2000d:03093)

19. Khoussainov, B., Nies, A., and Shore, R. Computable models of theories with few models. Notre Dame J. Formal Logic, 38(2), 1997, pp. 165-178. MR1489408 (99c:03049)

20. Miller, R. The $\Delta_{2}^{0}$-spectrum of a linear order. J. Symbolic Logic, 66, Issue 2, 2001, pp. 470-486. MR 1833459 (2002e:03065)

21. Melnikov, A. Enumerations and completely decomposable torsion-free abelian groups. Theory Comput. Syst., 45(4), 2009, pp. 897-916. MR2529752 (2010m:03069)

22. Slaman, T. Relative to any nonrecursive set. Proc. Amer. Math. Soc., 126, 1998, pp. 21172122. MR 1443408 (98h:03047)

23. Coles, R., Downey, R., and Khoussainov, B. On initial segments of computable linear orders. Order, 14, no. 2, 1997/98, pp. 107-124. MR.1631684(99m:03088)

24. Fuchs, L. Infinite abelian groups. Vol. II. Academic Press, 1973. MR0349869 (50:2362)

25. Wallbaum, J. Computability of algebraic structures. PhD thesis, University of Notre Dame, 2010. MR 2827319

26. Wehner, St. Enumerations, countable structures and Turing degrees. Proc. Amer. Math. Soc., 126, 1998, pp. 2131-2139. MR1443415 (98h:03059)

Department of Mathematics, Kazan Federal University, Kazan Tatarstan, Russia 420008

E-mail address: Iskander.Kalimullin@ksu.ru

Department of Computer Science, Private Bag 92019, University of Auckland, AuckLAND, NEW ZEALAND

E-mail address: bmk@cs.auckland.ac.nz

Department of Computer Science, Private Bag 92019, University of Auckland, AuckLAND, NeW ZEALAND

E-mail address: a.melnikov@cs.auckland.ac.nz 